Article

\title{
High-Throughput Phenotyping Approach for Screening Major Carotenoids of Tomato by Handheld Raman Spectroscopy Using Chemometric Methods
}

\author{
Hacer Akpolat 1,2®, Mark Barineau ${ }^{3}$, Keith A. Jackson ${ }^{3}$, Mehmet Z. Akpolat ${ }^{4}$, \\ David M. Francis ${ }^{5}$ (D), Yu-Ju Chen ${ }^{1}$ and Luis E. Rodriguez-Saona ${ }^{1, *}$ \\ 1 Department of Food Science and Technology, The Ohio State University, 110 Parker Food Science and \\ Technology Building, 2015 Fyffe Road, Columbus, OH 43210, USA; akpolat.1@osu.edu (H.A.); \\ chen.6599@buckeyemail.osu.edu (Y.-J.C.) \\ 2 Department of Nutrition and Dietetics, Bayburt University, 69000 Bayburt, Turkey \\ 3 Lipman Family Farms, 315 E New Market Road, Immokalee, FL 34142, USA; \\ Mark.Barineau@lipmanfamilyfarms.com (M.B.); Keith.Jackson@lipmanfamilyfarms.com (K.A.J.) \\ 4 Informatics Institute, Gazi University, 06500 Ankara, Turkey; zahid.akpolat@gmail.com \\ 5 Department of Horticulture and Crop Science, The Ohio State University, 119 Williams Hall, \\ 1680 Madison Avenue, Wooster, OH 44691, USA; francis.77@osu.edu \\ * Correspondence: rodriguez-saona.1@osu.edu
}

Received: 27 May 2020; Accepted: 28 June 2020; Published: 3 July 2020

check for updates

\begin{abstract}
Our objective was to develop a rapid technique for the non-invasive profiling and quantification of major tomato carotenoids using handheld Raman spectroscopy combined with pattern recognition techniques. A total of 106 samples with varying carotenoid profiles were provided by the Ohio State University Tomato Breeding and Genetics program and Lipman Family Farms (Naples, FL, USA). Non-destructive measurement from the surface of tomatoes was performed by a handheld Raman spectrometer equipped with a $1064 \mathrm{~nm}$ excitation laser, and data analysis was performed using soft independent modelling of class analogy (SIMCA)), artificial neural network (ANN), and partial least squares regression (PLSR) for classification and quantification purposes. High-performance liquid chromatography (HPLC) and UV/visible spectrophotometry were used for profiling and quantification of major carotenoids. Seven groups were identified based on their carotenoid profile, and supervised classification by SIMCA and ANN clustered samples with 93\% and $100 \%$ accuracy based on a validation test data, respectively. All-trans-lycopene and $\beta$-carotene levels were measured with a UV-visible spectrophotometer, and prediction models were developed using PLSR and ANN. Regression models developed with Raman spectra provided excellent prediction performance by ANN ( $\left.r_{\text {pre }}=0.9, \mathrm{SEP}=1.1 \mathrm{mg} / 100 \mathrm{~g}\right)$ and PLSR $\left(\mathrm{r}_{\text {pre }}=0.87, \mathrm{SEP}=2.4 \mathrm{mg} / 100 \mathrm{~g}\right.$ ) for non-invasive determination of all-trans-lycopene in fruits. Although the number of samples were limited for $\beta$-carotene quantification, PLSR modeling showed promising results $\left(\mathrm{r}_{\mathrm{cv}}=0.99\right.$, $\mathrm{SECV}=0.28 \mathrm{mg} / 100 \mathrm{~g}$ ). Non-destructive evaluation of tomato carotenoids can be useful for tomato breeders as a simple and rapid tool for developing new varieties with novel profiles and for separating orange varieties with distinct carotenoids (high in $\beta$-carotene and high in cis-lycopene).
\end{abstract}

Keywords: tomato carotenoids; handheld Raman spectroscopy; chemometrics; artificial neural networks

\section{Introduction}

Tomato fruit color is one of the most appealing characteristics for consumers in the market [1]. Carotenoid pigments are mainly responsible for the color of the skin and flesh of tomatoes providing yellow, orange, and red colors [2]. There is a growing interest in health benefits of different types of 
carotenoids because of their health promoting properties such as prevention of certain cancer types and cardiovascular diseases, reduction of age-related macular degeneration and improvement of eye health [3]. These health benefits are usually attributed to antioxidant properties of carotenoids and their interaction with free radicals [4]. Breeding for different tomato colors is a vigorous area of research because of consumer visual appeal and their attributed health benefits [5]. It has been shown that advancements in genetic technology can provide solutions for many specific purposes in plant breeding. However, utilizing the information provided by genetic technology is only possible when it is linked to phenotypic properties of the plant in a real-world setting [6].

High-performance liquid chromatography (HPLC) coupled with diode arrays or mass spectrometry (MS) detectors have been utilized for carotenoid analysis due to high precision, reproducibility and low detection limits [7]. UV-visible spectrophotometry is also extensively utilized for carotenoid analysis using the linear correlation between absorbance and concentration of carotenoids [3,8]. Other methods used less frequently for carotenoid analysis and profiling include matrix-assisted laser desorption ionization time-of-flight spectrometry (MALDI-TOF) [9], supercritical fluid chromatography coupled with mass spectrometry [10], and NMR [11]. However, none of these techniques are suitable for routine and in-field testing due to the cost and nature of the analysis (sample preparation, extraction of carotenoids, risk of degradation, etc.). Non-destructive methods can overcome the drawbacks of standard techniques, since no extraction and sample preparation is involved exposing carotenoids to degradation and/or isomerization, and can be utilized in the field providing true profile and level of the carotenoids [12].

Altering the levels and profiles of certain bioactive and nutritional compounds is possible through naturally occurring genetic variation [1]. However, the cost and labor of conventional methods of measuring these traits have limited characterization for breeding decisions [6]. A rapid and effective phenotyping method for carotenoids in the field would facilitate breeding selections, and tools are becoming possible with the developments of handheld sensors and computational approaches [13]. A rapid and non-destructive method could enhance the development and production of carotenoid-rich commodities to meet consumer demand for healthier foods [14].

Vibrational spectroscopy has received increased interest as a promising non-invasive and field-based high-throughput phenotyping platform providing rapid solutions in comparison to labor-intensive, time-consuming, costly, and laboratory-dependent traditional techniques. Infrared spectroscopy was reported by Rubio-Diaz et al. [15] as a rapid and cost-efficient method to profile tomato carotenoids. However, their method was destructive and required extraction of the lipid phase of the tomatoes. Raman spectroscopy is another fingerprinting technique that has shown success for carotenoid analysis, although there are limited reports, including the use of Raman chemical imaging to detect lycopene changes during ripening of tomatoes [7], spatially offset Raman spectroscopy to evaluate internal maturity of tomatoes [16], and resonance Raman spectroscopy for quantification of carotenoid compounds in fruits and vegetables [14].

Although handheld Raman spectroscopy has been tested for the detection of tomato ripening [17], it has not been tested before for the classification and quantification of different carotenoid profiles of tomatoes as a non-destructive method. Portable techniques have the advantage of bringing flexibility and rapidity to the location of analysis, which can be utilized by plant breeders, quality control personnel, or purchasing agents. Therefore, we aimed to test the feasibility of handheld Raman spectroscopy to discriminate and quantify the dominant carotenoids in tomatoes as a proof of concept for handheld Raman spectroscopy in the quality analysis of fruits and vegetables.

\section{Materials and Methods}

\subsection{Tomato Samples}

Tomato samples $(\mathrm{n}=106)$ with different carotenoid profiles and colors were obtained from the Ohio State University (Wooster, OH, USA), and Lipman Family Farms (Immokalee, FL, USA). 
There were 6 different colors of tomatoes with varying carotenoid profiles; red $(n=51)$, orange $(n=31)$, yellow $(n=10)$, green $(n=3)$, black/brown/purple $(n=7)$, pink $(n=4)$. Although the color of tomatoes is somewhat representative of the presence of carotenoids, the carotenoid profiles might be different for samples irrespective of visual coloration. For example, orange tomatoes can be obtained due the presence of high beta-carotene (beta), high tetra-cis-lycopene (tangerine), or ripening inhibitor alleles (rin or alc alleles). Similarly, pink and purple coloration in some tomatoes may be due to differences in anthocyanin pigments in the skin of the fruit due to colorless epidermis ( $y$ ) or anthocyanin fruit $(A f t)$ alleles. Alternatively, purple may be due to the retention of chlorophyll in green-flesh $(g f)$ tomatoes in the presence of trans-lycopene. Finally, the green-flesh gene combined with low carotenoid ( $r$ allele) result in fruit that are green at maturity. Therefore, assignment of carotenoid profiles and classes was made after HPLC data evaluation using retention times and spectrum of individual pigments.

\subsection{Raman Spectral Data Acquisition}

Tomatoes were washed with tap water to remove dirt from the surface and then dried with paper towels. The surface of the intact tomatoes was scanned using a handheld Raman spectrometer, Progeny $^{\mathrm{TM}}$ (Rigaku Analytical Devices, Wilmington, MA, USA). The handheld Raman spectrometer featured a 1064-nm excitation laser and thermoelectrically cooled InGaAs 512 pixel detector with a spectral range of $200-2500 \mathrm{~cm}^{-1}$ operating at $8 \mathrm{~cm}^{-1}$ spectral resolution. The laser power was set at $400 \mathrm{~mW}$ and exposure time was $10 \mathrm{~s}$ with 30 averages to maximize signal-to-noise ratio without damaging the sample. Three spectra were collected for each sample from three different points on the fruit surface (Figure 1). A background was collected after each sample spectrum collection.

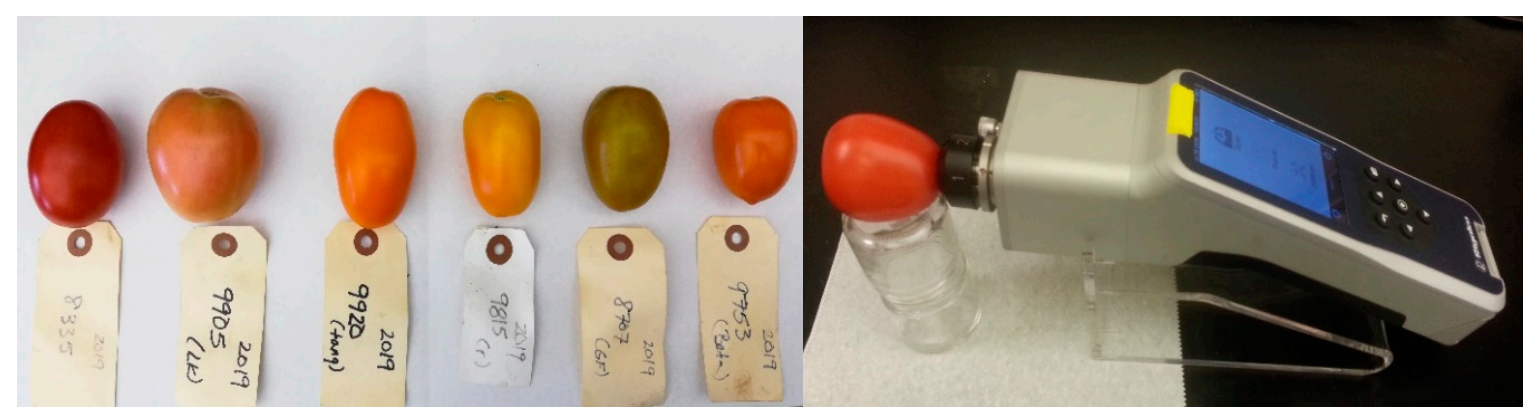

Figure 1. Tomato samples and surface scan using Rigaku's Progeny handheld Raman spectrometer.

\subsection{Profiling Carotenoids by HPLC}

After spectral collection, tomatoes were blended using a laboratory blender to be immediately extracted and analyzed by reverse phase high-performance liquid chromatography (HPLC). First, carotenoids were extracted using the method described by Anthon and Barrett [18] with slight modifications. An amount of $8 \mathrm{~mL}$ of hexane/ethanol/acetone solvent (HEA) 2:1:1 (v:v:v) was used as extraction solvent for $0.1 \mathrm{~g}$ sample. After vortexing for one minute, samples were stored for $20 \mathrm{~min}$ in the dark to prevent degradative effects of light and to allow for complete extraction. Following extraction, one $\mathrm{ml}$ of deionized water was added to separate phases. The samples were left for 10 more min in dark to allow a complete phase separation. For HPLC analysis, $3 \mathrm{~mL}$ of the hexane layer was transferred into amber glass tubes and the organic phase was removed by drying under nitrogen gas. Dried samples were dissolved in $3 \mathrm{~mL}$ of methanol/methyl tert-butyl ether (MTB) (1:1/v:v) solvent and filtered using Agilent 0.45- $\mu \mathrm{m}$ PTFE filters into amber HPLC vials.

Filtrated aliquots $(100 \mu \mathrm{L})$ were injected via autosampler into an Agilent HPLC (Agilent Technologies Inc., Santa Clara, CA, USA) equipped with a quaternary pump, a diode array detector (DAD), and a degasser. $\mathrm{A} \mathrm{C}_{30}$ column $(5 \mu, 250 \times 4.6 \mathrm{~mm})$ (YMC Inc., Allentown, PA, USA) was used for separation of different carotenoids during an 80-min run. HPLC conditions described by Rubio-Diaz et al. [15] were used to separate carotenoids. Mobile phases consisted of A: $81 \%$ methanol, 15\% MTBE, and 4\% 
water and B: 90\% MTBD, 7\% methanol, and 3\% water. A 75 min linear gradient run was used from 0 to $88 \% \mathrm{~B}$ at room temperature with a flow rate of $1.0 \mathrm{~mL} / \mathrm{min}$. Carotenoids show different peak shapes and maximum wavelength absorption according to the type of carotenoid in their UV-vis spectra [3]. Therefore, different wavelengths were used for determination of phytoene (290 nm), phytofluene (348 $\mathrm{nm})$, lutein, $\beta$-carotene, tetra-cis-lycopene, and $\delta$-carotene $(450 \mathrm{~nm})$, and all-trans-lycopene $(470 \mathrm{~nm})$. Tentative identification of carotenoids was performed based on retention times and specific UV-vis absorption spectrum of each compound. The analysis of HPLC chromatograms were made using ChemStation software (Agilent Technologies Inc., Santa Clara, CA, USA). HPLC data were used for class assignments of tomato samples by looking at common patterns in carotenoid profiles.

\subsection{Quantification of Carotenoids by UV-Visible Spectrophotometry}

Lycopene and $\beta$-carotene concentrations in tomatoes were determined using the method described by Anthon and Barrett [18] using the same extraction procedure as described in HPLC analysis section. A Cary 50 UV-vis spectrophotometer (Agilent Technologies Inc., Santa Clara, CA, USA) was used to perform absorbance measurements in triplicates. Concentrations of all-trans-lycopene and $\beta$-carotene were calculated using extinction coefficients and absorbance readings. The extinction coefficients used in this study were 172 and $139 \mathrm{mM}^{-1}$, and the maximum absorption wavelengths were 503 and $451 \mathrm{~nm}$ for lycopene and $\beta$-carotene, respectively. Our all-trans-lycopene dominant samples had many other minor carotenoids as well; however, these minor carotenoids have usually lower absorbance than all-trans-lycopene, so there is minimal interference by those at $503 \mathrm{~nm}$. On the other hand, $\beta$-carotene has a maximum absorption wavelength at $451 \mathrm{~nm}$ [18].

\subsection{Chemometric Analysis}

\subsubsection{Partial Least Square Regression (PLSR)}

Partial least squares regression (PLSR) is a quantitative technique for developing predictive models by combining features from multiple linear regression and principal component analysis (PCA). Usually it is useful when dependent variables were predicted using a large set of independent variables (predictors). The prediction of dependent variables is achieved by extracting orthogonal factors from predictors to obtain the best predictive power [19]. Since PLSR can analyze highly correlated data and a large number of variables, it is suitable for spectral data analysis [20]. Pirouette software (version 4.0, Infometrix Inc., Woodville, WA, USA) was used to create PLSR models for quantification of lycopene and $\beta$-carotene. Average of three spectra was used for each sample, since averaged spectra provided a better performance during model development. All spectral data was mean centered and Savitzky-Golay second derivative (15-point window) and smoothing were used to improve the quality of the spectral signal as they performed best among other pre-processing methods. Leave-one-out approach was used to perform internal cross-validation. Twenty percent of the samples from the whole data set was used as validation set to externally validate the models. Correlation coefficient of cross validation $\left(\mathrm{r}_{\mathrm{cv}}\right)$, correlation coefficient of prediction $\left(\mathrm{r}_{\mathrm{pred}}\right)$, standard error of cross validation (SECV), and standard error of prediction (SEP) were used to evaluate model performances. High correlation coefficients and low errors show a good prediction model [21]. Outliers were detected using outlier diagnostic tools of the software by evaluating the presence of any large or unusual residuals.

\subsubsection{Soft Independent Modelling of Class Analogy (SIMCA)}

Classification algorithms were built in Pirouette software (version 4.0, Infometrix Inc., Woodville, WA, USA) using the SIMCA method. SIMCA is a supervised classification technique, which allows clustering samples based on independent PCA conducted for each class separately [22]. Assignment of classes was based on HPLC data, and SIMCA was used to evaluate the ability of Raman spectroscopy to discriminate between different carotenoid profiles in tomatoes. For each sample, the average of three spectra was used for model development, since averaged spectra provided a better classification 
performance. All spectral data was mean centered and different data preprocessing techniques were applied to obtain best performances. Finally, standard normal variate (SNV) and Savitzky-Golay second derivative (25-point window) were selected to improve the quality of the spectral signal as they performed best in our dataset. SNV corrects the scattering effect normalizing each spectrum by standard deviation of responses across the entire spectral range [23]. Second derivative removes the non-linear background signal, which might be different for every sample [24]. Model performances were evaluated based on scores plot, discriminating power, and interclass distances (ICD). Scores plot was used for visualization of data showing the first three principle component (PC) and projections of data in a three-dimensional environment. Outlier diagnostics were performed using Mahalanobis distance and sample residual [25]. Discriminating power plot shows which region or wavenumber is most important for the separation of classes. ICD indicates how different classes from each other using residual standard deviations of all variables. A class distance over three suggests well-separated classes [22].

\subsubsection{Classification by Artificial Neural Networks (ANN)}

Artificial neural networks (ANN) is a nonlinear computational approach employed for classification, clustering, pattern recognition, and quantification purposes [26]. ANN mimics how a biological brain works with a network of neurons processing information and signals from neighboring neurons [27]. Many applications in the literature have been reported using ANN in analysis of various foods, including classification of red wines [28], maturity of dry-cured hams [29], classification of vegetable oils [30], evaluation of olive oil stability [31], classification of olive oils cultivars [32], detection of synthetic colorants [33], geographical traceability of bottled spring water [34], and classification of cow's milk [35] to name just a few. Advantages of ANN over linear chemometric methods includes its ability of dealing with non-linearity allowing for a better fit of the data, insensitivity to noise providing more accurate prediction, adaptability permitting the model to modify its structure accordingly for the changing environment, and generalization allowing to fit new data to the trained model [26].

In our study, ANN approaches were employed for classification and quantification modeling. Multilayer perceptron (MLP) feedforward approach [32] trained with a back-propagation (BP) algorithm was utilized to minimize the error by updating the network weights [28]. To prevent overfitting, we used dropout regularization technique [36] along with early stopping approach. For optimization, adaptive moment estimation (ADAM) was used with default parameters recommended by Kingma and $\mathrm{Ba}$ [37]. The neurons in the hidden layer were activated using rectified linear unit (ReLU) activation function, while Softmax function was used for the neurons of the output layer to compute the probability of classes [38]. Loss was calculated with categorical cross entropy (CCE) as an error function to measure the network performance [31]. Variable (spectral) selection was performed using a range scanning approach starting from the first input neuron (first wavelength of spectrum) to the last one with a sliding window (62-point) by moving forward two steps (wavelength) each time. The best spectral region was selected based on the lowest error rate obtained. The average of three spectra from each sample was used, and all spectral data was mean centered. Total number of trained models included different combinations of hyperparameters (i.e., dropout rate from 0 to 1 , number of hidden layers from 1 to 2, number of neurons from 2 to 250). Number of epochs for each trial was determined by the early stopping function, which prevents overfitting by stopping training once the model performance stops improving or begins to overfit [39]

We defined the Raman spectral data as "inputs" while the "output" consisted of the classes of carotenoids for classification or the concentration of the specific carotenoid for quantification. The data set was divided into a training set, a validation set for assessing the overfitting, and a test set for assessing the performance of the ANN model [34]. The samples were randomly divided into training:validation:test for ANN model using a 80\%:10\%:10\% structure for classification and a 60\%:20\%:20\% for quantification.

ANN model development was performed in Python using Keras (an open source modular neural network library). 


\section{Results}

\subsection{Characterization of the Carotenoid Profiles of Tomatoes}

The Raman spectra of selected red, tangerine, orange (high Beta-carotene), and yellow (low carotenoid) tomato breeding material are shown in Figure 2a. All Raman profiles showed common bands at $1007 \mathrm{~cm}^{-1}$ and $1158 \mathrm{~cm}^{-1}$ associated with $\mathrm{C}-\mathrm{CH}_{3}$ in-plane-rocking and C-C stretching, respectively. However, visual evaluation of Raman spectrum showed a shift at around roughly $1520 \mathrm{~cm}^{-1}$ corresponding to $\mathrm{C}=\mathrm{C}$ stretching depending on the major carotenoid of the tomato (Figure $2 \mathrm{~b}$ ). Tomatoes containing predominantly all-trans-lycopene showed a band maximum at $1519 \mathrm{~cm}^{-1}$, tomatoes having tetra-cis-lycopene and $\beta$-carotene showed a shift in the band centered at 1523 and $1528 \mathrm{~cm}^{-1}$, respectively. The low carotenoid samples did not show any significant carotenoid band in the Raman spectrum (Figure $2 b$ ). The position of the $C=C$ stretching Raman band was inversely related to the length of the conjugated polyene chain, isomerization (trans vs. cis), the side groups of polyene chain and the presence of other constituents bonded to carotenoids in the plant material [13,40-42].

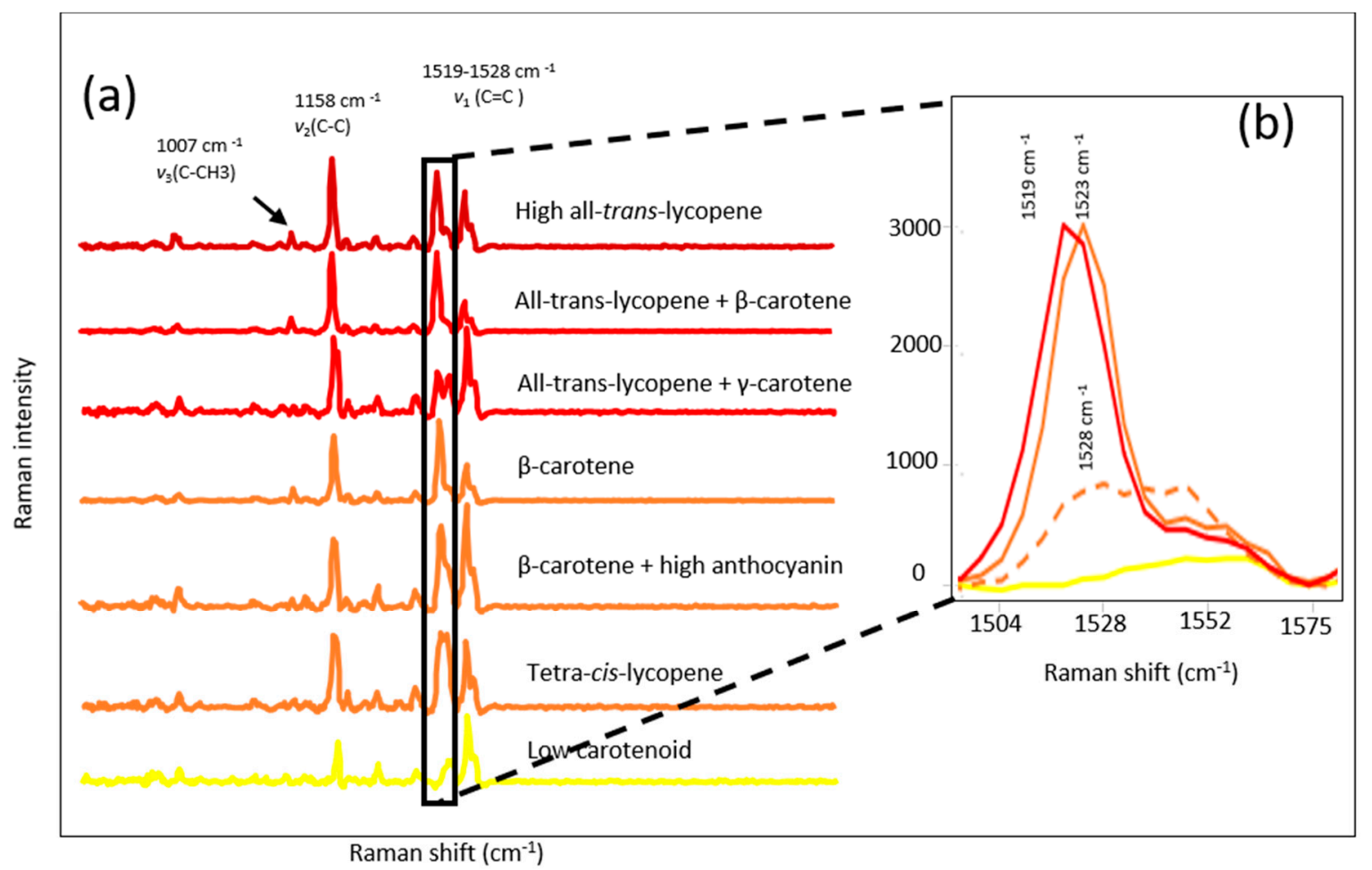

Figure 2. Raman spectra of different carotenoid profile tomatoes (a); colors are representative of fruit color with a few exceptions such as brown/black samples in " $\beta$-carotene + high anthocyanin group", and $v 1$ band shapes and locations for different class of tomatoes $(\mathbf{b})$; Red = all-trans-lycopene samples, solid orange $=\beta$-carotene, dashed orange $=$ tetra-cis-lycopene, yellow $=$ low carotenoid samples.

High performance chromatography was used for carotenoid profiling of the tomato breeding material including yellow, orange, red, and purple coloration. Four main groups were identified based on HPLC data including (1) all-trans-lycopene, (2) $\beta$-carotene, (3) tetra-cis-lycopene, and (4) low carotenoid samples carrying the $r$ gene (Figure 3 ). 


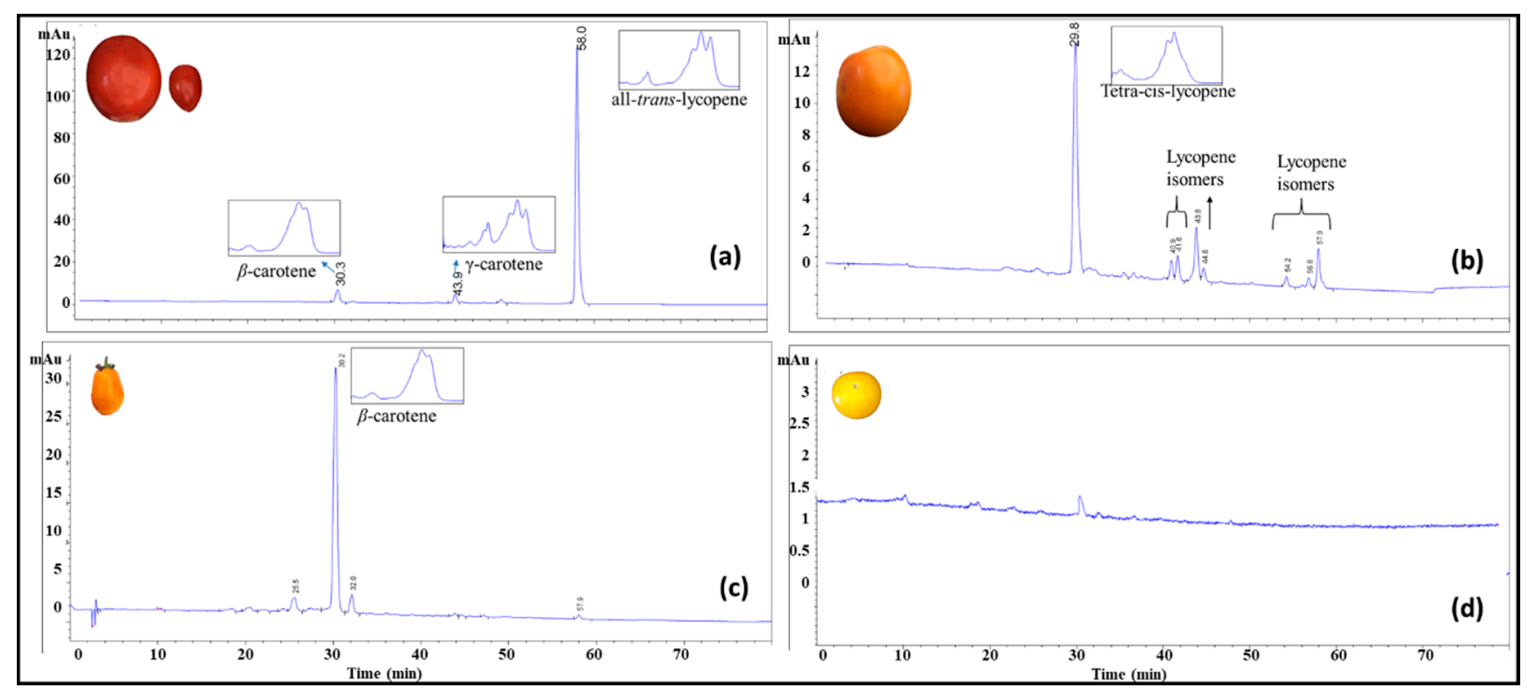

Figure 3. High-performance liquid chromatography (HPLC) chromatogram monitored at $450 \mathrm{~nm}$ and their corresponding absorption spectrum collected by the photodiode array detector for carotenoids in selected tomato breeding material. (a) Red tomatoes; (b) tangerine tomatoes high in tetra-cis-lycopene; (c) tangerine tomatoes high in $\beta$-carotene; (d) yellow tomatoes low in all carotenoid.

After the assignment of classes based on HPLC carotenoid profiles, pattern recognition algorithms were built using SIMCA. The spectra were preprocessed before analysis by using standard normal variate (SNV) and Savitzky-Golay second derivative filtering (25-point window) to remove scattering noise effects and correct for baseline drift. The SIMCA model was able to separate the groups based on the information in the $1370-1700 \mathrm{~cm}^{-1}$ region and revealed seven clusters instead of the four suggested by HPLC. These seven different groups were based on their dominant carotenoid profile as well as the presence of other carotenoids or anthocyanins resulting in the following classes: (1) high levels of all-trans-lycopene and no $\beta$-carotene, (2) all-trans-lycopene and $\beta$-carotene, (3) all-trans-lycopene and $\gamma$-carotene, (4) $\beta$-carotene (and low lycopene), (5) $\beta$-carotene and anthocyanins, (6) tetra-cis-lycopene, and (7) low in all carotenoids. The class projections (Figure 4) of the seven groups showed well separated classes in a three-dimensional environment using the first three principal components (PC). Interclass distances (ICD) values ranged from 3.4 to 38.5. The highest ICD (38.5) was between low carotenoid and the all-trans-lycopene $+\beta$-carotene group, while lowest ICD (3.4) was between tetra-cis-lycopene and the all-trans-lycopene (high) group. Our study showed that all ICD values between classes were $>3$ (Table 1) with significant separation of clusters [22].

The discriminating power graph in the SIMCA model shows the wavelengths mainly responsible for the separation of the groups [22], which can be representative of specific chemical structures. The band at $1523 \mathrm{~cm}^{-1}$ was responsible for separation of different groups in the discriminating power graph. This explains that the wavelength shift caused by predominant carotenoids of tomatoes was mainly responsible for separation, since $1523 \mathrm{~cm}^{-1}$ corresponds to the area for the $v_{1}$ band at around $1519-1530 \mathrm{~cm}^{-1}$ depending on the carotenoid type. We validated our classification model using a separate data set $(20 \%$ of the whole data set) to evaluate prediction performance. The external validation revealed that the SIMCA classification model had a $93 \%$ accuracy for the prediction of independent samples. 


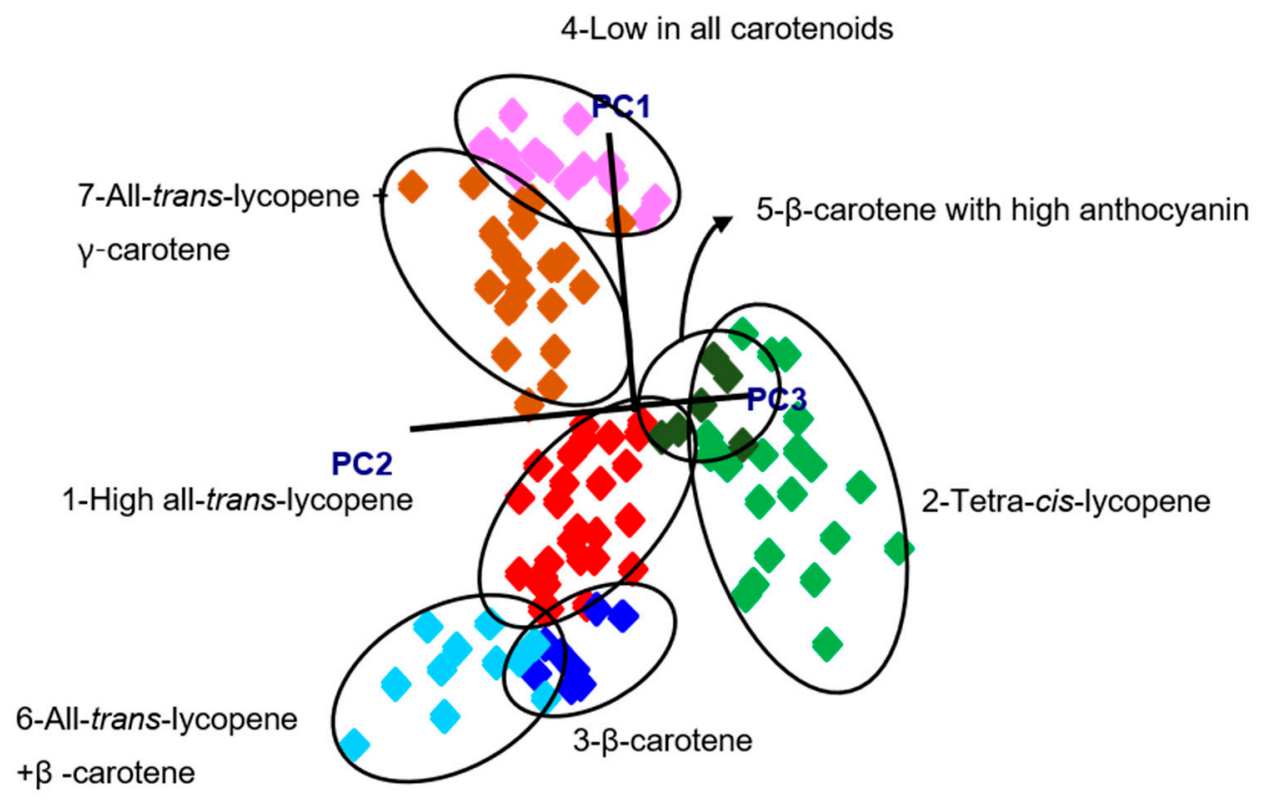

Figure 4. Soft independent modelling of class analogy (SIMCA) 3D class projections using the information in the $1370-1670 \mathrm{~cm}^{-1}$ region.

Table 1. SIMCA inter-class distances among four groups.

\begin{tabular}{|c|c|c|c|c|c|c|c|}
\hline & $\begin{array}{l}\text { All-Trans- } \\
\text { Lycopene } \\
\text { (High) }\end{array}$ & $\begin{array}{l}\text { Tetra-Cis- } \\
\text { Lycopene }\end{array}$ & $\beta$-Carotene & $\begin{array}{c}\text { Low } \\
\text { Carotenoids }\end{array}$ & $\begin{array}{l}\beta \text {-Carotene } \\
+ \text { High } \\
\text { Anthocyanin }\end{array}$ & $\begin{array}{c}\text { All-Trans- } \\
\text { Lycopene + } \\
\beta \text {-Carotene }\end{array}$ & $\begin{array}{l}\text { All-Trans- } \\
\text { Lycopene } \\
\text { (Low) }\end{array}$ \\
\hline $\begin{array}{c}\text { all-trans-lycopene } \\
\text { (high) }\end{array}$ & 0.0 & 3.4 & 6.1 & 18.4 & 5.7 & 7.5 & 6.2 \\
\hline tetra-cis-lycopene & & 0.0 & 6.9 & 8.7 & 4.7 & 10.7 & 7.1 \\
\hline$\beta$-carotene & & & 0.0 & 34.9 & 12.9 & 3.8 & 19.5 \\
\hline low carotenoids & & & & 0.0 & 9.9 & 38.5 & 5.4 \\
\hline $\begin{array}{c}\beta \text {-carotene with } \\
\text { high anthocyanin }\end{array}$ & & & & & 0.0 & 17.0 & 6.3 \\
\hline $\begin{array}{l}\text { all-trans-lycopene } \\
+\beta \text {-carotene }\end{array}$ & & & & & & 0.0 & 20.9 \\
\hline $\begin{array}{c}\text { all-trans-lycopene } \\
\text { (low) }\end{array}$ & & & & & & & 0.0 \\
\hline
\end{tabular}

Additionally, we investigated ANN as a non-linear method for classification of tomato carotenoids based on handheld Raman spectroscopy using the class assignments from SIMCA algorithms. ANN models using the full spectrum were not able to separate classes very well, so variable selection was important for the predictive performance by eliminating uninformative variables and noise [43]. The range from 1377 to $1671 \mathrm{~cm}^{-1}$ gave the lowest error and highest accuracy and was used as input $(n=60)$ for the network. This range was very similar to the range used in SIMCA analysis and included the band around $1520 \mathrm{~cm}^{-1}$ corresponding to $\mathrm{C}=\mathrm{C}$ stretching band shift of carotenoids.

Among 1000 trials to find the optimum hyperparameters of the model, 100\% accuracy was reached for 35 different ANN models. The highest accuracy (100\%) and minimum test loss (0.03804) was obtained with a 0.4 dropout rate using 242 neurons in one hidden layer. The eight most efficient networks were selected based on the highest accuracy obtained for the test set and the lowest test loss (Table 2). The properties were given in Table 2 arranged in a decreasing order of test loss. Test loss was calculated with CCE loss function; the lower the test loss means the higher the probability for a particular class assignment [44]. The number of epochs shown in the table was quite different for each model because of the early stopping function, which stops the training process once the network performance starts deteriorating to prevent overfitting. Similar classification abilities were obtained with different network topologies and hyperparameters. 
Table 2. The most efficient 8 networks selected from 2000 networks based on classification accuracy and test loss.

\begin{tabular}{ccccc}
\hline Network Topology * & Dropout & Epoch & Classification Accuracy (\%) & Test loss (CCE) \\
\hline $60-242-7$ & 0.4 & 814 & 100 & 0.03804 \\
$60-178-7$ & 0.1 & 738 & 100 & 0.05484 \\
$60-240-7$ & 1 & 746 & 100 & 0.06449 \\
$60-106-7$ & 1 & 1417 & 100 & 0.06616 \\
$60-199-7$ & 0.4 & 852 & 100 & 0.07257 \\
$60-226-7$ & 0.1 & 499 & 100 & 0.07568 \\
$60-201-7$ & 0.7 & 1388 & 100 & 0.07896 \\
$60-238-7$ & 0.4 & 712 & 100 & 0.089558 \\
\hline
\end{tabular}

* number of neurons in input layer, hidden layer, and output layer, respectively.

\subsection{Quantification of All-Trans-Lycopene and $\beta$-Carotene by PLSR and ANN}

Among 106 samples, 58 had all-trans-lycopene, and 17 had $\beta$-carotene as the dominant carotenoid based on HPLC results. Quantification of all-trans-lycopene and $\beta$-carotene was performed using UV-Vis spectrophotometer, and concentration ranged from 1.8 to $21.5 \mathrm{mg} / 100 \mathrm{~g}$ for all-trans-lycopene and 1.6 to $9.5 \mathrm{mg} / 100 \mathrm{~g}$ for $\beta$-carotene. PLSR was used to correlate spectral and reference analysis data using the Savitzky-Golay second derivative filtering with a window size of 15 and smoothing that removed the effect of variation in the baseline [45]. PLSR models were generated using a calibration $(80 \%)$ and external validation $(20 \%)$ data sets. Cross-validation (leave-one-out) of the calibration set selected four latent variables that explained most of the variance in the data and avoided overfitting, giving a correlation coefficient of and standard error of cross-validation of $1.4 \mathrm{mg}$ lycopene $/ 100 \mathrm{~g}$ (Figure 5). By using the external validation set, the PLSR model gave prediction performances of 0.87 for correlation coefficient and a standard error of prediction (SEP) of $2.3 \mathrm{mg}$ lycopene/100 g sample. The only report to our knowledge available for lycopene quantification in tomatoes using a handheld Raman spectrometer $(1064 \mathrm{~nm})$ has showed an $R^{2}$ of 0.88 in their regression model, with no error value or external validation data set reported [46]. Sheng et al., [47] reported on a non-destructive method for lycopene determination in cherry tomatoes, with a lycopene concentration of $7.51 \pm 1.27 \mathrm{mg} / 100 \mathrm{~g}$, using portable NIR spectroscopy, reporting a correlation coefficient of 0.80 and SEP of $0.74 \mathrm{mg} / 100 \mathrm{~g}$. Our data set had a wider lycopene range and comprised different types of tomatoes, including round, Roma, cherry, and grape tomatoes.
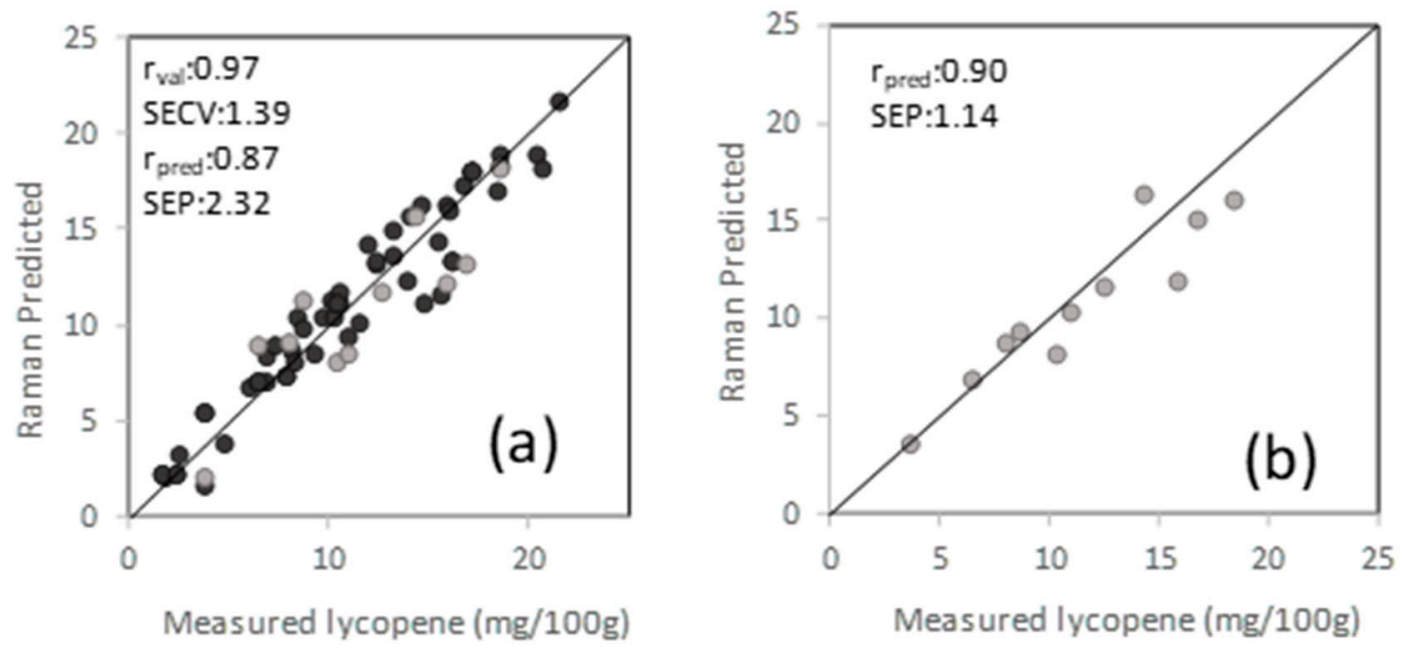

Figure 5. Regression model for all-trans-lycopene quantification for PLS (a) and artificial neural network (ANN); (b) black representing calibration set, and gray representing external validation test set. 
The PLSR prediction algorithms built for $\beta$-carotene were also promising with $\mathrm{r}_{\mathrm{cv}}$ of 0.99 and SECV of 0.28 ; however, the number of samples available was limited $(n=17)$. Therefore, further investigation is necessary with more samples.

The feedforward MLP ANN was used to correlate Raman spectral data and carotenoid reference values to predict all-trans-lycopene content of tomatoes. Since the number of $\beta$-carotene samples was very limited, an ANN model was not developed for $\beta$-carotene. Data was divided into three groups; training $(60 \%)$, validation $(20 \%)$, and test $(20 \%)$ sets. The same spectral region $\left(1377-1671 \mathrm{~cm}^{-1}\right) \mathrm{was}^{2}$ used as in the classification model. Dropout regularization technique, ADAM optimization technique was used as described in the previous sections. ReLU activation function was used in both hidden and output layers. The best MLP model and optimum hyperparameters of the model were obtained with trial and error. The best performing ANN model included 219 of neurons in a hidden layer with a dropout rate of 0.5 and an epoch cycle of 1000 . Regression coefficient for test data was found as 0.90 with an RMSE value of $1.14 \mathrm{mg} / 100 \mathrm{~g}$ for quantification of all-trans-lycopene.

\section{Conclusions}

Handheld Raman spectroscopy combined with different pattern recognition techniques allowed for the non-destructive classification of carotenoid profiles in tomatoes based on dominant carotenoid types. Raman spectroscopy allows for time-efficient (less than $5 \mathrm{~min}$ ), user-friendly, and in-field analysis of carotenoids and our green approach allows carotenoids to be analyzed in their natural environment avoiding the risk of isomerization and degradation during the extraction process. Classification of tomatoes based on their dominant carotenoid profile were promising by using SIMCA and ANN pattern recognition techniques. SIMCA showed a classification accuracy of $93 \%$ when an independent data set was tested for validation of the model, while ANN provided $100 \%$ classification accuracy in the test data set. The same spectral information was used for quantification of all-trans-lycopene by using PLSR and ANN modeling giving good regression coefficients $>0.9$ and standard error of prediction (SEP) of 2.3 and $1.1 \mathrm{mg} / 100 \mathrm{~g}$ for PLSR and ANN, respectively. The improved predictive performance of ANN could be attributed to its ability to deal with non-linear functions.

Our findings fill the gap in the literature for a non-destructive and portable technique for profiling and quantifying major tomato carotenoids utilizing the strong laser penetration ability of Raman spectroscopy for in-situ and non-destructive fruit analysis. The technology can provide breeders real-time information in improving tomato varieties based on unique carotenoid profiles and colors.

Author Contributions: Conceptualization, H.A., Y.-J.C., and L.E.R.-S.; investigation, H.A.; resources, D.M.F., M.B., and K.A.J.; writing-original draft preparation, H.A.; writing-review and editing, D.M.F., and L.E.R.-S.; supervision and funding acquisition, L.E.R.-S.; data analysis, H.A., M.Z.A., and L.E.R.-S. All authors have read and agreed to the published version of the manuscript.

Funding: This work was financially supported by Lipman Family Farms (Naples, FL, USA), and samples were provided by Lipman Family Farms (Naples, FL, USA) and Ohio State University Tomato Breeding and Genetics program.

Conflicts of Interest: The authors declare no conflict of interest.

\section{References}

1. Pek, Z.; Helyes, L.; Gyulai, G.; Foshee, W.; Daood, H.; Lau, J.; Waters, L.j. Molecular Profiling-Fruit Carotenoids Components of Six American Heirloom Tomatoes (Solanum lycopersicum). J. Forensic Biomech. 2016, 7. [CrossRef]

2. Borguini, R.G.; da Silva Torres, E.A.F. Tomatoes and tomato products as dietary sources of antioxidants. Food Rev. Int. 2009, 25, 313-325. [CrossRef]

3. Kopec, R.E.; Cooperstone, J.L.; Cichon, M.J.; Schwartz, S.J. Analysis Methods of Carotenoids. Anal. Antioxid. Rich Phytochem. 2012, 4, 105-148. [CrossRef] 
4. Luterotti, S.; Marković, K.; Franko, M.; Bicanic, D.; Madžgalj, A.; Kljak, K. Comparison of spectrophotometric and HPLC methods for determination of carotenoids in foods. Food Chem. 2013, 140, 390-397. [CrossRef] [PubMed]

5. Saltveit, M.E. Fruit Ripening and Fruit Quality. In Tomatoes; Heuvelink, E., Ed.; CABI Publishing: Wallingford, UK, 2005.

6. Furbank, R.T.; Tester, M. Phenomics—technologies to relieve the phenotyping bottleneck. Trends Plant Sci. 2011, 16, 635-644. [CrossRef]

7. Qin, J.; Chao, K.; Kim, M.S. Investigation of Raman chemical imaging for detection of lycopene changes in tomatoes during postharvest ripening. J. Food Eng. 2011, 107, 277-288. [CrossRef]

8. Dzakovich, M.P.; Gas-Pascual, E.; Orchard, C.J.; Sari, E.N.; Riedl, K.M.; Schwartz, S.J.; Francis, D.M.; Cooperstone, J.L. Analysis of Tomato Carotenoids: Comparing Extraction and Chromatographic Methods. J. Aoac Int. 2019, 102, 1069-1079. [CrossRef]

9. Fraser, P.D.; Enfissi, E.M.A.; Goodfellow, M.; Eguchi, T.; Bramley, P.M. Metabolite profiling of plant carotenoids using the matrix-assisted laser desorption ionization time-of-flight mass spectrometry. Plant. J. 2007, 49, 552-564. [CrossRef]

10. Matsubara, A.; Bamba, T.; Ishida, H.; Fukusaki, E.; Hirata, K. Highly sensitive and accurate profiling of carotenoids by supercritical fluid chromatography coupled with mass spectrometry. J. Sep. Sci. 2009, 32, 1459-1464. [CrossRef]

11. Tiziani, S.; Schwartz, S.J.; Vodovotz, Y. Profiling of carotenoids in tomato juice by one- and two-dimensional NMR. J. Agric. Food Chem. 2006, 54, 6094-6100. [CrossRef]

12. Schulz, H.; Baranska, M.; Baranski, R. Potential of NIR-FT-Raman spectroscopy in natural carotenoid analysis. Biopolymers 2005, 77, 212-221. [CrossRef] [PubMed]

13. Araus, J.L.; Cairns, J.E. Field high-throughput phenotyping: The new crop breeding frontier. Trends Plant Sci. 2014, 19, 52-61. [CrossRef] [PubMed]

14. Bhosale, P.; Ermakov, I.V.; Ermakova, M.R.; Gellermann, W.; Bernstein, P.S. Resonance Raman quantification of nutritionally important carotenoids in fruits, vegetables, and their juices in comparison to high-pressure liquid chromatography analysis. J. Agric. Food Chem. 2004, 52, 3281-3285. [CrossRef] [PubMed]

15. Rubio-Diaz, D.E.; De Nardo, T.; Santos, A.; de Jesus, S.; Francis, D.; Rodriguez-Saona, L.E. Profiling of nutritionally important carotenoids from genetically-diverse tomatoes by infrared spectroscopy. Food Chem. 2010, 120, 282-289. [CrossRef]

16. Qin, J.; Chao, K.; Kim, M.S. Nondestructive evaluation of internal maturity of tomatoes using spatially offset Raman spectroscopy. Postharvest Biol. Technol. 2012, 71, 21-31. [CrossRef]

17. Trebolazabala, J.; Maguregui, M.; Morillas, H.; de Diego, A.; Madariaga, J.M. Portable Raman spectroscopy for an in-situ monitoring the ripening of tomato (Solanum lycopersicum) fruits. Spectrochim. Acta Part A 2017, 180, 138-143. [CrossRef]

18. Anthon, G.; Barrett, D.M. Standardization of a rapid spectrophotometric method for lycopene analysis. Acta Hortic. 2007, 758, 111-128. [CrossRef]

19. Abdi, H. Partial least squares regression and projection on latent structure regression (PLS Regression). Wiley Interdiscip. Rev. 2010, 2, 97-106. [CrossRef]

20. Wold, S.; Sjöström, M.; Eriksson, L. PLS-regression: A basic tool of chemometrics. Chemom. Intell. Lab. Syst. 2001, 58, 109-130. [CrossRef]

21. Kvalheim, O.M.; Karstang, T.V. SIMCA—Classification by Means of Disjoint Cross Validated Principal Components Models. Data Handl. Sci. Technol. 1992, 9, 209-248. [CrossRef]

22. Duckworth, J. Mathematical Data Preprocessing. In Near-Infrared Spectroscopy in Agriculture; Al-Amoodi, L., Ed.; American Society of Agronomy, Inc.; Crop Science Society of America, Inc.; Soil Science Society of America, Inc.: Madison, WI, USA, 2004; pp. 115-132.

23. Wold, S.; Sjöström, M. SIMCA: A Method for Analyzing Chemical Data in Terms of Similarity and Analogy; ACS Publications: Washington, DC, USA, 1977; pp. 243-282. [CrossRef]

24. Olivieri, A.C. Mathematical Pre-processing. In Introduction to Multivariate Calibration; Springer: Cham, Switzerland, 2018; pp. 139-158. [CrossRef] 
25. Brereton, R.G. Multivariate Pattern Recognition in Chemometrics: Illustrated by Case Studies. Available online: https://www.sciencedirect.com/bookseries/data-handling-in-science-and-technology/vol/9 (accessed on 20 June 2020).

26. Debska, B.; Guzowska-Świder, B. Application of artificial neural network in food classification. Anal. Chim. Acta 2011, 705, 283-291. [CrossRef] [PubMed]

27. Ballabio, D.; Todeschini, R. Multivariate classification qualitative analysis. In Infrared Spectroscopy for Food Quality Analysis and Control; Elsevier Inc.: Amsterdam, The Netherlands, 2009; Available online: https: //pdfs.semanticscholar.org/b6bd/4d48199287fefb932c8dc1fb9d69686bf082.pdf (accessed on 20 June 2020).

28. Díaz, C.; Conde, J.E.; Estévez, D.; Pérez Olivero, S.J.; Pérez Trujillo, J.P. Application of multivariate analysis and artificial neural networks for the differentiation of red wines from the Canary Islands according to the island of origin. J. Agric. Food Chem. 2003, 51, 4303-4307. [CrossRef] [PubMed]

29. Prevolnik, M.; Andronikov, D.; Žlender, B.; Font-i-Furnols, M.; Novič, M.; Škorjanc, D.; Čandek-Potokar, M. Classification of dry-cured hams according to the maturation time using near infrared spectra and artificial neural networks. Meat Sci. 2014, 96, 14-20. [CrossRef] [PubMed]

30. Tanajura da Silva, C.E.; Filardi, V.L.; Pepe, I.M.; Chaves, M.A.; Santos, C.M.S. Classification of food vegetable oils by fluorimetry and artificial neural networks. Food Control. 2015, 47, 86-91. [CrossRef]

31. Silva, S.F.; Anjos, C.A.R.; Cavalcanti, R.N.; Celeghini, R.M.D.S. Evaluation of extra virgin olive oil stability by artificial neural network. Food Chem. 2015, 179, 35-43. [CrossRef]

32. Binetti, G.; Del Coco, L.; Ragone, R.; Zelasco, S.; Perri, E.; Montemurro, C.; Schena, F.P. Cultivar classification of Apulian olive oils: Use of artificial neural networks for comparing NMR, NIR and merceological data. Food Chem. 2017, 219, 131-138. [CrossRef]

33. Benvidi, A.; Abbasi, S.; Gharaghani, S.; Dehghan Tezerjani, M.; Masoum, S. Spectrophotometric determination of synthetic colorants using PSO-GA-ANN. Food Chem. 2017, 220, 377-384. [CrossRef]

34. Gutiérrez-Reguera, F.; Jurado, J.M.; Montoya-Mayor, R.; Ternero-Rodríguez, M. Geographical classification of Spanish bottled mineral waters by means of iterative models based on linear discriminant analysis and artificial neural networks. Neural Comput. Appl. 2018, 29, 459-468. [CrossRef]

35. Behkami, S.; Zain, S.M.; Gholami, M.; Khir, M.F.A. Classification of cow milk using artificial neural network developed from the spectral data of single- and three-detector spectrophotometers. Food Chem. 2019, 294, 309-315. [CrossRef]

36. Srivastava, N.; Hinton, G.; Krizhevsky, A.; Sutskever, I.; Salakhutdinov, R. Dropout: A Simple Way to Prevent Neural Networks from Overfitting. J. Mach. Learn. Res. 2014, 15, 1929-1958.

37. Kingma, D.P.; Ba, J. Adam: A Method for Stochastic Optimization. arXiv 2014, arXiv:1412.6980.

38. Ichinohe, Y.; Yamada, S.; Miyazaki, N.; Saito, S. Neural network-based preprocessing to estimate the parameters of the X-ray emission of a single-temperature thermal plasma. Mon. Not. R. Astron. Soc. 2018, 475, 4739-4744. [CrossRef]

39. Chen, L.; Huang, J.F.; Wang, F.M.; Tang, Y.L. Comparison between back propagation neural network and regression models for the estimation of pigment content in rice leaves and panicles using hyperspectral data. Int. J. Remote Sens. 2007, 28, 3457-3478. [CrossRef]

40. De Oliveira, V.E.; Castro, H.V.; Edwards, H.G.; de Oliveira, L.F.C. Carotenes and carotenoids in natural biological samples: A Raman spectroscopic analysis. J. Raman Spectrosc. 2010, 41, 642-650. [CrossRef]

41. Vijayalakshmi, K.; Jha, A.; Dasgupta, J. Ultrafast Triplet Generation and its Sensitization Drives Efficient Photoisomerization of Tetra-cis-lycopene to All-trans-lycopene. J. Phys. Chem. B 2015, 119, 8669-8678. [CrossRef] [PubMed]

42. Llansola-Portoles, M.J.; Pascal, A.A.; Robert, B. Electronic and vibrational properties of carotenoids: From in vitro to in vivo. J. R. Soc. Interface 2017, 14, 20170504. [CrossRef] [PubMed]

43. Spiegelman, C.H.; McShane, M.J.; Goetz, M.J.; Motamedi, M.; Yue, Q.L.; Coté, G.L. Theoretical Justification of Wavelength Selection in PLS Calibration: Development of a New Algorithm. Anal. Chem. 1998, 70, 35-44. [CrossRef]

44. Ramesh, S.; Vydeki, D. Recognition and classification of paddy leaf diseases using Optimized Deep Neural network with Jaya algorithm. Inf. Process. Agric. 2019. [CrossRef]

45. Brown, C.D.; Vega-Montoto, L.; Wentzell, P.D. Derivative preprocessing and optimal corrections for baseline drift in multivariate calibration. Appl. Spectrosc. 2000, 54, 1055-1068. [CrossRef] 
46. Hara, R.; Ishigaki, M.; Kitahama, Y.; Ozaki, Y.; Genkawa, T. Excitation wavelength selection for quantitative analysis of carotenoids in tomatoes using Raman spectroscopy. Food Chem. 2018, 258, 308-313. [CrossRef] [PubMed]

47. Sheng, R.; Cheng, W.; Li, H.; Ali, S.; Akomeah Agyekum, A.; Chen, Q. Model development for soluble solids and lycopene contents of cherry tomato at different temperatures using near-infrared spectroscopy. Postharvest Biol. Technol. 2019, 156, 110952. [CrossRef]

C 2020 by the authors. Licensee MDPI, Basel, Switzerland. This article is an open access article distributed under the terms and conditions of the Creative Commons Attribution (CC BY) license (http://creativecommons.org/licenses/by/4.0/). 